\title{
Adaptive Business Protocol Support in Service Oriented B2B Collaborations (Extended Abstract)
}

\author{
Adomas Svirskas, Justinas Bedžinskas, Bob Roberts, Ioannis Ignatiadis, Michael Wilson \\ Vilnius University, Kingston University London, CCLRC Rutherford Appleton Laboratory \\ adomas@svirskas.com, justinas.bedzinskas@mif.vu.lt, r.roberts@kingston.ac.uk, \\ i.ignatiadis@kingston.ac.uk,m.d.wilson@rl.ac.uk
}

\begin{abstract}
Service Oriented Architecture approach in general and the Web services technology in particular are becoming more and more important in modern networked organisations and Virtual Organisations (VO). However, it is not enough to have the business functionality of the partners packaged as (Web) services; there is also a need for business-aligned order of interaction between these services a.k.a. business protocols, which can also be reused. This paper discusses business protocol adaptivity aspects and suggests some ideas how to support the changes in the business protocols in implementations of the partners' services (end-points).
\end{abstract}

\section{Introduction}

The Web services, as the main realisation of the SOA [2] paradigm, imply a significant integration potential at both application and business process levels. These services, being homogeneous in terms of their functional description, advertising mechanisms and the protocols needed to use them, do not suffer from the usual middleware-level interoperability problems. On the other hand, the services encapsulate business-aligned discreet functionality through their interfaces, making B2B integration tasks easier.

Interactions between Web services and their clients in order to achieve a common goal are called conversations [1]. These conversations need to be governed by some unambiguous rules, specifying which conversations are valid and what kind of data at what point is properly understood by the services. The rules are specified as part of the business protocol supported by the service (where the word 'business' is used to differentiate it from a communication protocol) [3]. Business protocols are examples of why a simple interface description is not enough in Web services. In fact, to completely describe a service, it is necessary to specify not only its interface but also the business protocols that the service supports.

The business protocols, however, are subject to frequent changes and their capability to adapt is crucial for success of participating businesses, which, in turn, need to respond fast to the protocols' changes. In this paper we present an idea of adaptive business protocol support through dynamic processing of Web services choreography at the partners' end-points and flexible mapping of choreographed message exchanges to the local processes and components.

\section{The background}

In the specification of the business protocols there are two aspects that need particular consideration. On the one hand, there is a need to capture the commonality of business rules and the interaction scenarios together with the semantics of business data to be used in the conversations. On the other hand, these protocols need to be described in some declarative machine-readable form for all the prospective collaboration partners to agree upon collectively and enact individually at run-time.

Business protocols imply peer to peer service interaction model, in this case it is important to describe the sequence and the format of message exchange between the collaboration participants. Such descriptions are known as choreographies. RossTalbot [7] defines choreography as a description of the peer to peer externally observable interactions that exist between services. The interactions are described from a global or neutral point of view and not from any one services perspective. Choreography description can be used to generate the necessary behavioural contract for each of the peers and to verify programmatically that all the separate cases (success, failure) of a business scenario are consistent with each other. 
Currently there are two major ongoing (coordinated) efforts in choreography modelling and description standardisation. One of them is the OASIS ebXML Business Process (ebBP) Technical Committee (TC) working on ebBP Specification v 2.0.1 [5], another the W3C WS Choreography Working Group, finalising the specification of Web Services Choreography Description (WS-CDL) Language [4]. Our work is mostly related to the WSCDL effort due to better support of the Web services technology; however we base some of our ideas on the ebBP work as well.

\section{The approach and future research}

Our paper aims to contribute to the aspect of adaptivity of business protocol support by collaborating partners. This aspect is a part of a broader issue of adaptivity in the business collaboration domain. In the context of B2B collaborations adaptivity has several flavours: adaptivity of the business models to different business requirements and environments (Hofreiter \& Huemer discuss this in [6]), adaptivity of business protocols in response to business models' changes, adaptivity of the partners' end-point services to the changes in the business protocols descriptions. In addition, the business protocols should be adaptive to the changes of the partners enacting the roles defined in the protocols (both choreography and orchestration should support this).

Once the choreography definition is created, it needs to be deployed publicly and advertised for reuse. Then it needs to be (re)distributed to (or perhaps, depending on the overall interaction model, discovered by) the appropriate collaboration participants (dynamically chosen to play certain applicable roles) and accepted by each of them as a contract for subsequent interactions. The choreography script is then enacted by the participants' end-point services and this process should be monitored and managed by user and administrative tools.

This scenario correlates with the adaptivity aspects and support of commonly agreed business protocols helps to raise the overall adaptivity level by promoting reuse of these protocols. However, compliance of the participating services to a common contract might result in the choreography enactment being hard coded into the implementations of the services and/or their composition mechanisms. This reduces reusability of the services and makes it difficult to change choreography description without a need for massive programmatic changes at the participating end-points.
Therefore, some intelligence is needed to allow smoother propagation of the protocol changes. In our work we propose to introduce an application-level gateway between the public business protocol (choreography) and the end-point composite services. This gateway is a part of the implementations of the collaboration participants' end-points. The functions of the gateway range from virtualisation of the business services of the end-point to choreography support via role-based decomposition of it and mapping of the incoming messages to the appropriate processing entities within the end-point.

Choreography support service, the core part of the gateway mediates two-way message exchange between the "outside world" and the local processing entities. This service takes a choreography definition, the role which the end-point is supposed to play and maps the choreography messages to local operations at run-time. Therefore, the incoming messages can be routed, translated, and processed by the business services. In addition, dynamic selection of processing entities to play the prescribed roles, policy enforcement, trust and security support and other non-functional tasks can be performed by the handlers.

Our current work and future research is aimed at designing and efficient gateway architecture and choreography support service including choreography description interpretation, message processing and flexible operation mapping.

\section{References}

[1] F. Casati, "Web services conversations: why do they matter and what they mean to you”, Presentation, HP Labs, 2004

[2] H. He, "What is Service-Oriented Architecture?"

[3] G. Alonso, F. Casati, H. Kuno, and V. Machiraju, Web Services Concepts, Architectures and Applications, Springer-Verlag, 2004.

[4] WS-CDL, Web Services Choreography Description Language Version 1.0. W3C Candidate Recommendation, 2005

[5] ebBP, ebXML Business Process Specification Schema Technical Specification v2.0.1. Public Review Final Draft r03, 2 January 2006.

[6] B. Hofreiter \& C. Huemer, "Registering a Business Collaboration Model in Multiple Business Environments”, Proceedings of the OTM Workshop on Modeling Inter-Organizational Systems (MIOS 2005) Larnaca, Cyprus, 2005

[7] S. Ross-Talbot, "Orchestration and Choreography: Standards, Tools and Technologies for Distributed Workflows”, NETTAB Workshop - Workflows management: new abilities for the biological information overflow, Naples, Italy, 2005. 